\title{
Using standard image compression algorithms to store data from computational fluid dynamics is
}

\author{
Jörg Schmalzl* \\ Institut für Geophysik, Universität Münster, Corrensstr. 24, D-48149 Münster, Germany
}

Received 17 October 2001; received in revised form 4 March 2003; accepted 10 March 2003

\begin{abstract}
Three-dimensional numerical modeling of fluid flows is an important research tool to understand many fluid dynamical effects observed in nature. With the strong growth of available computational resources the use of such models has greatly increased over the last years. Because the available mass storage has not increased in the same order as the CPU speed many researchers nowadays face the problem of how to store and transfer the large data sets produced by the model calculations for post-processing. The use of lossy wavelet-based compression techniques on this data has been investigated in several publications. These techniques are often specialized to one problem and are not easy to implement. In the area of digital media, however, advances have been made for still image (JPEG, JPEG-2000) and motion image (MPEG) compression. In this paper we investigate the usefulness of these image compression algorithms for the storage of data from computational fluid dynamics on regular cartesian grids. We analyze both the compression ratios achieved and the error introduced by these lossy compression schemes. We found that, for our purposes, the JPEG compression scheme allows an easy-to-use, portable, robust, and computationally efficient lossy compression. For the easy use of these compression algorithms we present a simple wrapper library.
\end{abstract}

(C) 2003 Elsevier Ltd. All rights reserved.

Keywords: Computational fluid dynamics; Data compression; Visualization; Post-processing

\section{Introduction}

Due to the rapid increase of available computer power over the last years, the use of fully three-dimensional (3D) numerical simulations of nonstationary fluid flows has greatly increased. Many examples come from geophysical research: flows in porous media, creeping convection in the Earth's mantle and magneto-convection in the outer core. Other prominent examples are local and global atmospheric models and ocean circulation models. Only a decade ago the use of such models was worldwide limited to a few meteorological services who had the computational resources whereas today such calculations are feasible on a cluster of work-

\footnotetext{
${ }^{2 / 2}$ Code on server at http://www.iamg.org/CGEditor/index. htm.

*Tel.: + 49-251-833-4703; fax: +49-251-833-6100.

E-mail address: joergs@uni-muenster.de (J. Schmalzl).
}

stations or a large computer. The increased efficiency of the numerical models is due not only to the availability of faster computers, but also to the development of sophisticated numerical methods such as multi-grid methods or adaptive grid refinement.

This together has led to a revival of the classical field of fluid dynamics where fundamental questions could not be addressed analytically due to the nonlinear nature of the governing equations and laboratory experiments were difficult to quantify.

Numerical models work in a way that they solve a number of coupled partial differential equations (PDEs) on a computational grid using a numerical method such as Finite Differences, Finite Volumes, Finite Elements or Spectral Methods. The amount of output data ranges from three variables per computational node when solving a Navier-Stokes equation for an incompressible media to eight variables when looking at magnetoconvection in a compressible flow. Often there is also 
interest in monitoring the transport of a varying number of chemical components, such as trace gases in atmospheric models, which adds another advection-diffusion equation to the numerical model.

The spatial resolution of such models is today typically in the range from $32 \times 32 \times 32$ grid-nodes up to $256 \times 256 \times 64$ grid-nodes. Because of the complex nature of the evolving flows, one often has to integrate the equations in time for several thousand timesteps in order to observe generic behavior and to analyze the different timescales involved.

Although computers have become faster such a simulation cannot be done interactively but will still require computer time in the order of weeks or even months. Visualization and analysis of the results is typically done by post-processing the data saved at different stages of the numerical simulation. Because it is not always obvious which fraction of the computed data is needed for post-processing, the researcher tries to save as much data as possible.

Moreover due to specialized graphics hardware the machine used to post-process the data is often different from the one used to carry out the simulation. Especially when using a mainframe computer this often means that the binary format and/or the byte order of the machines differ. This is not a principal problem, but it poses constraints on the way the data are stored.

Storing the result of one time-step with a resolution of $128 \times 128 \times 64$ nodes and four variables per node leads to a filesize of 17 Mega Bytes (MB) when using a 32-bit binary floating point value and about $50 \mathrm{MB}$ when using a portable ASCII representation. For calculating trajectories in the flow during post-processing or generating an animated image sequence of the data, one typically needs to save every 10th Lewy-Courant constrained timestep. Given that the calculation often consists of several thousand timesteps, the resulting data filesize is often in the order several Gigabytes (GB). This makes storage and handling of the data not only inconvenient, but also makes it impossible to keep several result files on a disk when carrying out a systematic parameter study.

Therefore it is highly desirable to store the data in a more compact manner. Standard lossless compression algorithms such as Huffman encoding or the LempelZiv encoding (e.g. Salomon, 2000) which are implemented for example in the gzip utility do lead to a size reduction of only $10-20 \%$ for the binary data and are computationally intensive.

For post-processing the data calculated in the fluiddynamical simulation it is often acceptable that the data are not available in full precision but with a lower accuracy. The required accuracy depends on the kind of post-processing carried out on the data. For visualization purposes, for example, a relatively low precision is sufficient.
Another important aspect when thinking about data compression is the existence of characteristics in the data set which could aid the compression process. Examples of these are temporal and/or spatial coherent structures or the fact that fields do change smoothly in space due to diffusive terms in the governing equations. When the compression scheme chosen is able to utilize these characteristics a high compression ratio can be achieved.

The field of data compression is still evolving at a high rate. Because of its key relevance for different fields there are many publications available. The probably most complete reference is the book by Salomon (2000) which treats all of the compression algorithms applied in the present paper. Application of data compression to Earth science can be found for seismic data (Vassiliou and Wickerhauser, 1997; Villasenor et al., 1996; Lervik et al., 1996) and for ocean-model data (Tao and Moorhead, 1994a, b; Bradley and Brislawn, 1993). All these publications apply wavelet-based methods. Their aim is mostly to speed up visualization and exploration of data. In the field of turbulence simulation Zubair et al. (1992) and Wilson (2002) also discuss the use of waveletbased methods.

These publications present the leading edge in data compression. Their main aim is to achieve the highest compression ratios while minimizing the introduced artifacts. The approach of the present paper is a different, more technical one. For many researchers involved in CFD, particularly in small and medium sized research groups, the size of the data generated is becoming a real problem. In this case the priorities are different. The key issues are: The robustness of the method, the availability of the source code, the portability of the source code over different machine architectures, the computational resources required, and the programming effort required for using this method. In addition it is important to have a wide range of compression ratios easily available in order to account for different post-processing needs. All these issues are for most researchers more important than the optimal compression ratio.

In this paper we address most of the issues mentioned before by using standard video and image compression software which has mostly been available for years. Lossy encoding schemes are widely used in still and motion image compression. The compression algorithms used in these fields are complex and have undergone extensive testing. This poses the question we address in the paper of whether they could also be used to store the data from fluid-dynamical simulation in an effective manner. Codes like the JPEG implementation has been available for over a decade and have been ported to a wide variety of different architectures. With the help of a small wrapper library we have made the different standard compression algorithms easily available to both Fortran $77 / 90$ and $\mathrm{C} / \mathrm{C}++$ programs. 
In Section 2 we will present a simple approach by storing each data point as two ASCII characters and discuss the error in doing so. In the next section we use the standard JPEG picture compression library with a 12-bit accuracy to store the data. The new wavelet-based JPEG-2000 approach to data-compression is investigated in Section 4. The following section applies the same investigation to a MPEG-2 compression algorithm. A comparison of the compression methods discussed is presented in Section 6. In Section 7 we present a user friendly set of subroutines which makes it easy to access the algorithms discussed. In the conclusions we will discuss possible future development.

\section{The data sets used for testing}

For testing the different data-compression algorithms we used different data sets from numerical simulation of convection in a highly viscous media. Such a flow is described by the continuity equations for mass, momentum and energy. Because we only investigate incompressible flows we can introduce a vector streamfunction which implicitly fulfils the continuity equation. In our limit of infinite Prandtl number the toroidal component of the streamfunction $\left(A_{z}\right)$ is zero. The numerical code used to carry out the simulations (Houseman, 1990) solves the governing equations by applying a mixed spectral-finite-differences approach. Due to the streamfunction formulation we have three variables per computational node, namely the temperature $T$ and the two streamfunction components $A_{x}$ and $A_{y}$. For testing we used calculations carried out with different resolution, vigour of convection and boundary conditions. The first data set, data $64 \times 64 \times 64$ describes convection in a square container with a Rayleighnumber of $R a=6 \times 10^{6}$ with stress-free boundary conditions for the velocity. The spatial resolution is $64 \times 64 \times 64$ nodes which is reflected by the name. The second test-case, data256 $\times 256 \times 32$, describes convection in a rectangular tank with an aspect ratio of eight by eight by one. The velocity boundary conditions are rigid in this case which leads to small-scale structures. The third test case data256×256×64 has again stress-free velocity boundary conditions, a Rayleigh number of $R a=5 \times 10^{6}$ and an aspect ratio of four by four by one. A snapshot of the internal temperature structure of the data $256 \times 256 \times 64$ case is presented in Fig. 1 . The plot shows two temperature isosurfaces at the non-dimensional temperatures of 0.3 and 0.7 . The temperature field is characterized by very thin thermal boundary layers at the top and bottom of the box and by strongly localized up- and down-wellings. Superimposed on the major upand down-wellings are boundary layer instabilities which are drifting with the large scale flow. We have also used a down-sampled version of this data set $($ data $128 \times 128 \times 32)$ in order to limit computational effort in some cases.

\section{Using two characters for data storage}

Using this method we first determine the maximum and minimum value of a data array for each variable. Maximum and minimum values are stored in the file and the array is mapped into an array of unsigned 16-bit

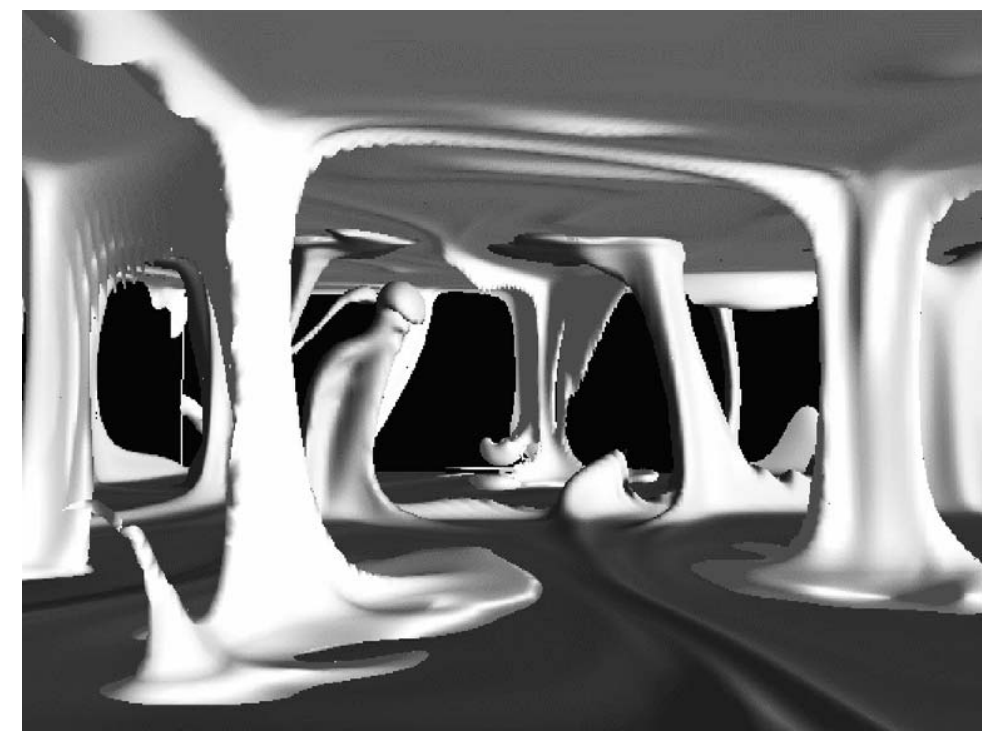

Fig. 1. Visualization of internal temperature distribution in data set data $256 \times 256 \times 64$. Two temperature isosurfaces at nondimensional temperatures of $T=0.3$ and 0.7 indicating position and shape of thin thermal up- and down-wellings. 
integers [0-65535]. The integer data can be written to a file as two ASCII characters thus avoiding portability problem due to different byte order. The maximum error for this method is equal to $1 / 2^{16} \times 100=0.0015 \%$ of the difference between the maximum and minimum value of the data array stored. The error can be further reduced by rounding the data appropriately when compressing. The compression step is therefore:

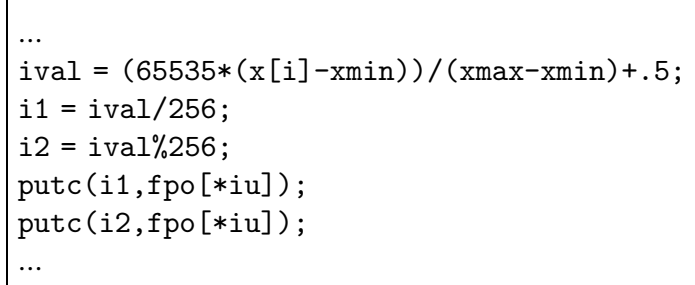

The resulting data file is $50 \%$ of the size of binary written 32-bit data-file and about $15 \%$ of the size of a formatted written file. Like the formatted written file this data is portable over different processor architectures.

In practical terms, the use of the 16-bit data storage proved to be accurate enough for most post-processing purposes. Especially since many measures taken in fluid dynamics are non-local the error seems to be negligible. The compression scheme does not require any extra memory and the processor time spent on converting the data is more than balanced by having to read/write only half the amount of data to disk.

\section{Using the JPEG picture compression library}

The JPEG standard is a widely used still image compression algorithm. ${ }^{1}$ It supports compression of both color and grayscale images. Greatly simplified, the algorithm for compressing grayscale images works in a way that it calculates discrete cosine transforms of $8 \times 8$ blocks of the data. Pending on the choice of a compression parameter it neglects a certain number of the smallest Fourier coefficients, and finally stores the remaining coefficients using a Huffman-Coding algorithm. As one can envisage, this works especially well for smooth images where the high wavenumber coefficients are small. Since the data from fluid-dynamical calculations are smooth because of both momentum and heat diffusion, we expected that using the JPEG compression algorithm would make use of the feature in order to achieve high compression rates. For our purpose we are using the freely available implementation from the Independent JPEG Group (see footnote 1). We are using

\footnotetext{
${ }^{1}$ Wallace, G.K., 1991. Independent JPEG Group example implementation ftp://ftp.uu.net/graphics/jpeg.
}

the version $6 \mathrm{a}$ in this paper. The JPEG standard allows either 8-bit or 12-bit data precision which is implemented in the library as a compile time option. We decided to use the 12-bit version for our purposes and compressed the data as a single component grayscale image. Because JPEG introduces a header which contains a compression table for every picture, optimal compression ratios are achieved when using large pictures. Therefore we store the 3D data set as a set of 2D images. The fluid dynamical data sets is first scaled to an integer array $\left[0,2^{12}-1\right]$. This step introduces an error of $1 / 2^{12} \times 100=0.0244 \%$ of the difference between the maximum and minimum value of the data array stored. This array is subsequently compressed by the JPEG library as one picture of the size $x$ dim, ydim $\times$ zdim. The compression ratios achieved by this method are presented in Fig. 2. The rate of compression is dependent on the compression parameter of the JPEG library which can be varied between 0 and 100 which controls how many of the Fourier coefficients will be neglected. The logarithmic $y$-axis indicates the size of the data sets relative to the size of the binary written data set in $\%$. Because the efficiency of the compression depends both on the dimensions of the compressed data set and on the contents of the data we used three different data set from numerical simulation of convection which differ both in size and internal structure of the flow. The size of the resulting data set varies between $1 \%$ of the original size for a compression parameter setting of 0 and $20 \%$ for a compression parameter setting of 100 . The data set data $256 \times 256 \times 32$ where the flow has most internal structure compresses worst and the best compression is achieved with the large data256 $\times 256 \times 64$ data set. Although the compression ratios for the different data set do differ significantly all three data sets exhibit the same dependence on the compression factor. We further note that functional dependence of the compression on the compression parameter does have a relatively low slope in the interval $q=[15,85]$. The next question to be answered is the error introduced in the data set by this lossy compression scheme. In Fig. 3, we present both the maximum and the averaged error and its dependence on the compression parameter for the three different data sets. In order to determine the maximum and averaged error a binary data set is compressed and decompressed using the algorithm and the resulting data-values $\left(x\right.$ comp $\left._{i}\right)$ are compared with the original values of the data set $\left(\right.$ xorig $\left._{i}\right)$ where $i$ is the index of the variable ranging from 1 to the number of nodes $(n)$. The maximum error is calculated as

$e r r_{\max }=\max _{i}\left(\mid\right.$ xorig $_{i}-$ xcomp $\left._{i} \mid\right)$

and the averaged error is defined as

err $_{a v}=\frac{1}{n} \sum_{i} \mid$ xorig $_{i}-$ xcomp $_{i} \mid$. 


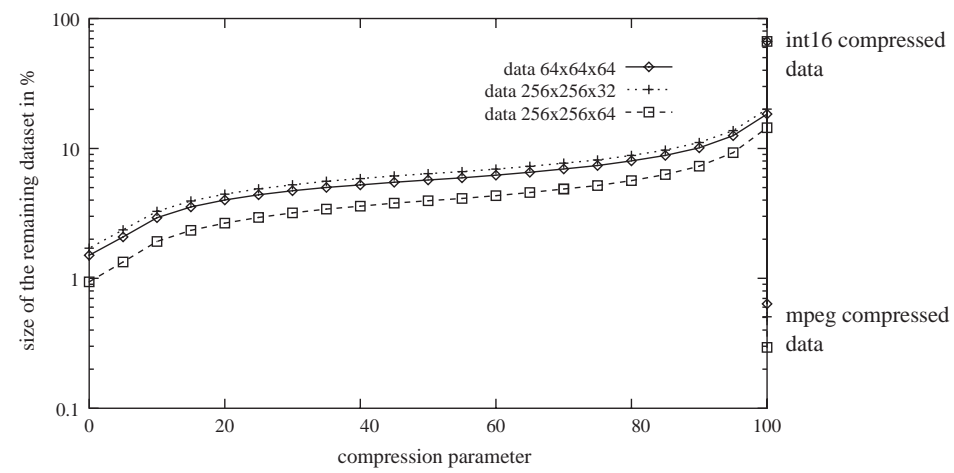

Fig. 2. Size of remaining data set in percent compared to binary written data set in dependence of compression parameter. Please note that $y$-axis has a logarithmic scale. We have used three different data sets with different size and different internal structure as denoted by different symbols. On right-hand border of plot we have added compression factors for Integer16 compression and MPEG compression scheme.

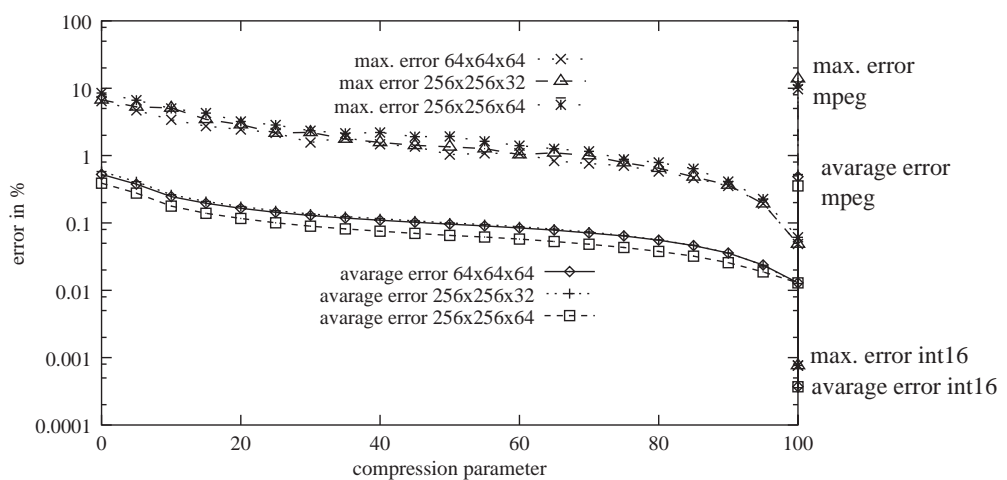

Fig. 3. Maximum and average error for different compression schemes. $y$-axis has as in Fig. 2 a logarithmic scale. On $x$-axis we have plotted compression parameter for JPEG compression. On right-hand border values for Integer 16 and MPEG compression are shown.

Both the maximum and the averaged error decrease with increasing compression parameter. It is also interesting to note that the difference between the averaged and the maximum error is about a factor of 20 which is different from the previous discussed integer encoding where only a factor of two was observed as indicated by the symbols on the right-hand side of Fig. 3. The spatial location and distribution of the introduced error is presented in Fig. 4. When investigating the compressed data set we found that the maximum errors occurred in regions with high gradients. Artifacts introduced by the $8 \times 8$ blocking of the JPEG algorithm are visible for very strong compression. In Table 1, we give exact numbers for the compression and the errors occurring. For a compression parameter of $q=85$ we found a maximum error of $0.63 \%$ and an averaged error of $0.032 \%$. Timing the efficiency of the read and write routines is complicated because it depends heavily on the system configuration used and on the buffering performed by the system. Therefore we have used a system (SGI
Origin 200 with 1 GB main memory) where all read/write operations are performed into memory only. This is done by first writing the data and immediately reading the data into the program again. On the one hand this approach gives reliable numbers whereas in the case where disk $\mathrm{i} / \mathrm{o}$ is involved the timings varied greatly. On the other hand, this timing favors uncompressed $\mathrm{i} / \mathrm{o}$ since the time for read/writing large data set to/from disk (which should be to first order, proportional to the size of the data set) does not contribute to the timings. The time needed to compress the data or uncompress the JPEG compressed data set was found to be relatively insensitive to the compression factor chosen. As documented in Table 1 the time needed to uncompress the data set is about half the time needed for the compression step. The timings are comparable to the Integer 16 compression and about a factor of 10 slower than for the binary written data. Given the overall time needed for the numerical simulation the extra time needed for compressing the data is negligible. 


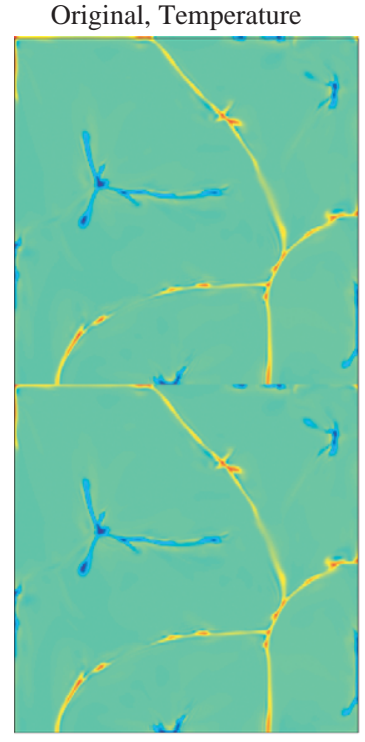

(a)

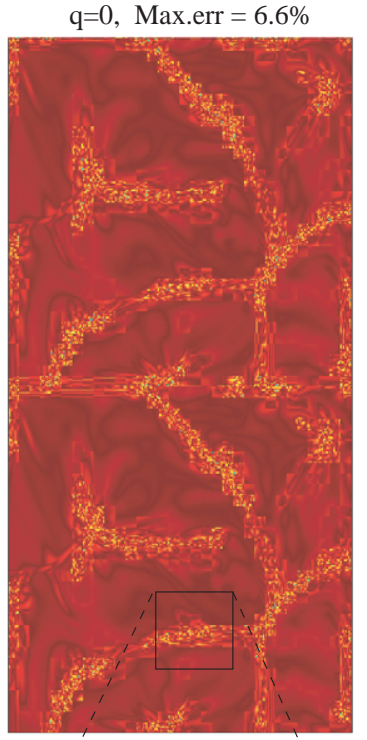

(b)

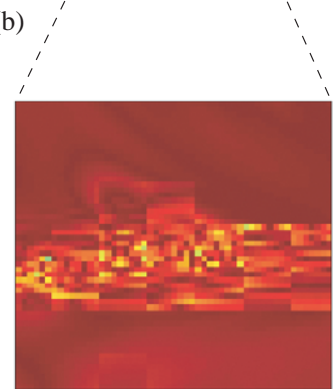

$\mathrm{q}=80$, Max. $\mathrm{err}=0.68 \%$

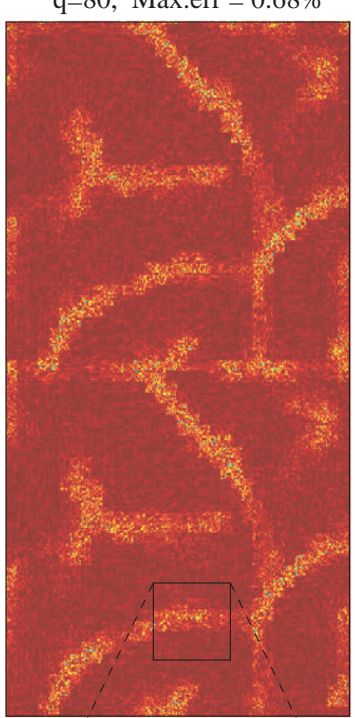

(c)

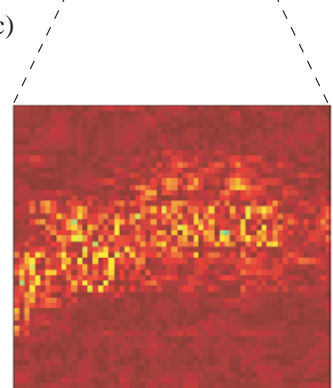

$\mathrm{q}=100$, Max.err $=0.045 \%$

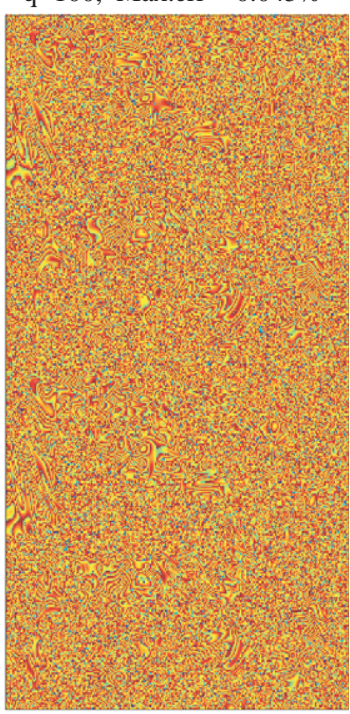

(d)

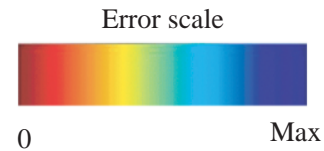

Fig. 4. In (a) two $x-y$ slices of temperature field for a resolution of $256 \times 256 \times 64$ nodes at a $z$-value of 32 and 33 are shown. Clearly gradients are related to up- and down-wellings. In (b)-(d) spatial distribution of error generated by JPEG compression is plotted for different values of compression parameter $q$. For $q=0$ and 80 high errors are correlated with position of strong gradients in temperature field. For highly compressed case (b) $(q=0)$ it is also possible to identify blocking artifacts introduced by $8 \times 8$ block structure of JPEG algorithm. For lowest compression $(q=100)$ no Fourier modes are truncated and error is generated only by quantization to 12-bit.

Table 1

Comparison of different storage methods for a $256 \times 256 \times 64$ CFD data set with three variables per node

\begin{tabular}{lllllll}
\hline Data set: $256 \times 256 \times 64$ & ASCII (E12.6) & Binary & Integer16 & JPEG $q=85$ & JPEG-2000 & MPEG \\
\hline Filesize & $172 \mathrm{MB}$ & $51 \mathrm{MB}$ & $25 \mathrm{MB}$ & $2400 \mathrm{kB}$ & $2200 \mathrm{kB}$ & $113 \mathrm{kB}$ \\
Max. error \% & 0.000006 & 0 & 0.00076 & 0.64 & 0.5 & 11.09 \\
Average error \% & 0.000002 & 0 & 0.00037 & 0.032 & 0.02 & 0.35 \\
Write time (s) & 114.9 & 0.85 & 7.79 & 9.26 & 58. & 33.1 \\
Read time (s) & 9.98 & 0.62 & 6.13 & 5.77 & 4.7 \\
\hline
\end{tabular}

\section{The JPEG-2000 compression algorithm}

JPEG-2000 ISO/IEC (2000) is a new standardised algorithm for still image compression that has good chances to replace the JPEG standard. Different from the standard JPEG image compression it is based on the discrete wavelet transform (DWT). The DWT is dyadic and can be performed either by the reversible Le Gall$(5,3)$ taps filter (Gall and Tabatabai, 1988) for lossless coding or with the non-reversible Daubechies- $(9,7)$ taps biorthogonal (Antonini et al., 1992) filter which provides higher compression but is lossy. Describing the complete compression algorithm is beyond the purpose of this paper, and is presented elsewhere (see references in 
ISO/IEC 2000; Santa-Cruz and Ebrahimi, 2000). We have used the JASPER sample implementation version $0.72^{2}$ of the JPEG-2000 standard for compressing the fluid dynamical data sets. The software can be used to compress gray-scale images with both 8- and 16-bit precision per data point. Fig. 5a shows the compression ratio for 8- and 16-bit JPEG-2000 compression using the Daubechies- $(9,7)$ wavelet. For comparison we have also plotted the 12-bit JPEG compression. It can be seen that the 16-bit compression approaches for high quality a size of $20 \%$ of the binary (32-bit) data set. This shows that even for the highest quality there is still a considerable compression compared with the 16-bit data as presented in Section 3. The curve for the 8-bit data flattens out at lower values of the compression factor. In Fig. $5 b$ we have plotted both the maximum and the averaged error caused by the compression. Due to the different integer accuracy (16-, 12-, and 8-bit) of the compressed data they do converge to different maximum and averaged errors at high compression values. Interesting to note is that the difference between the maximum and the averaged error is over most of the parameter range less than one order of magnitude for the JPEG-2000 compression but more than one order of magnitude for the JPEG compression. It is also important for practical purposes to investigate the time needed to compress and decompress the data set (Fig. 5c). The wavelet based method for 16-bit does consume approximately an order of magnitude more compute time than the JPEG-based method for both compression and decompression. Different from the JPEG-based method the time needed for compression/ decompression of the data set depends on the compression factor-highly compressed data sets do take less time than less compressed (i.e. high accuracy) data sets.

Since the compression parameter is differently defined for the JPEG and the JPEG-2000 compression we have chosen to plot the dependency of the size of the compressed data set on the error connected with the compression in Fig. 6. In this figure we also show the influence of the two different base Wavelets on the compression process. The Le Gall- $(5,3)$ taps filter leads to a higher accuracy on the cost of a bigger data file. For lower accuracy the Daubechies-(9,7) filter leads to better compression. What is interesting in this figure is however that for moderate compression, where a maximum error of $1 \%$ is acceptable, all the three graphs are close both for average and maximum error. This is in contradiction to comparisons between the compression schemes carried out using real world images (Santa-Cruz and Ebrahimi, 2000) where the JPEG-2000 performed much better. We attribute this to the smoothness of the field, which suits especially the JPEG compression.

\footnotetext{
${ }^{2}$ Adams, M.D., 2000. THE JASPER PROJECT HOME PAGE, http://www.ece.ubc.ca/mdadams/jasper.
}

\section{Using the MPEG-1 compression algorithm}

Different from the JPEG compression which is a still image compression scheme the MPEG-1 standard aims at compressing series of images normally being a movie. The whole compression algorithm is complex and is described in Legall (1991). Since in such movies normally only parts of the image change from one frame to another the MPEG-1 compression relies on storing a base image completely and then only store the changes to this base frame for a certain number of frames. This approach could be also interesting for storing data from fluid dynamical calculations since we often do observe large scale structures such as the up and down wellings. The existence of these structures could be used when storing the data of one time step as a series of slices. The software MPEG-1 decoder/encoder we are using ${ }^{3}$ does require the input to be a series of 8-bit images describing the three component in the YUV colorspace. In order to achieve optimal compression the two chrominance components UV are stored only at half the image resolution whereas the luminance component is stored at full resolution. For our datacompression we used the luminance component only. The data from the fluid dynamical experiments are scaled first to an integer array $[0,255]$ and subsequently piped through the MPEG-1 compressor. The compression rates achieved by this approach are amazingly high. As indicated in Fig. 2 they can be as high as $99.7 \%$ compared to the size of the binary data set. But also the maximum error generated is high and can be in the order of $10 \%$ as shown in Fig. 3. The averaged error is much smaller however and is in the range of $0.3-0.5 \%$. MPEG-1 compression is compute intensive which is documented in Table 1. The compression of the data set takes about five times longer than the JPEG compression. The decompression step however takes a comparable amount of time to the JPEG compression. This seems to be important as the data is normally only compressed once when the data is generated but decompressed many times when post-processing the data. Moreover the post-processing and visualization is often done in an interactive manner where fast loading of the data is important.

\section{Comparison of the different compression schemes}

The four lossy compression schemes presented here all have the advantage of being portable over different computer architectures. In terms of compression ratio and accuracy they are different. On the one end the Integer 16 compression accurately reproduces the data

\footnotetext{
${ }^{3}$ Berkely, MPEG-1 decoder/encoder ftp://havefun.stanford. edu/pub/mpeg/MPEGv1.2.1.tar.Z.
} 

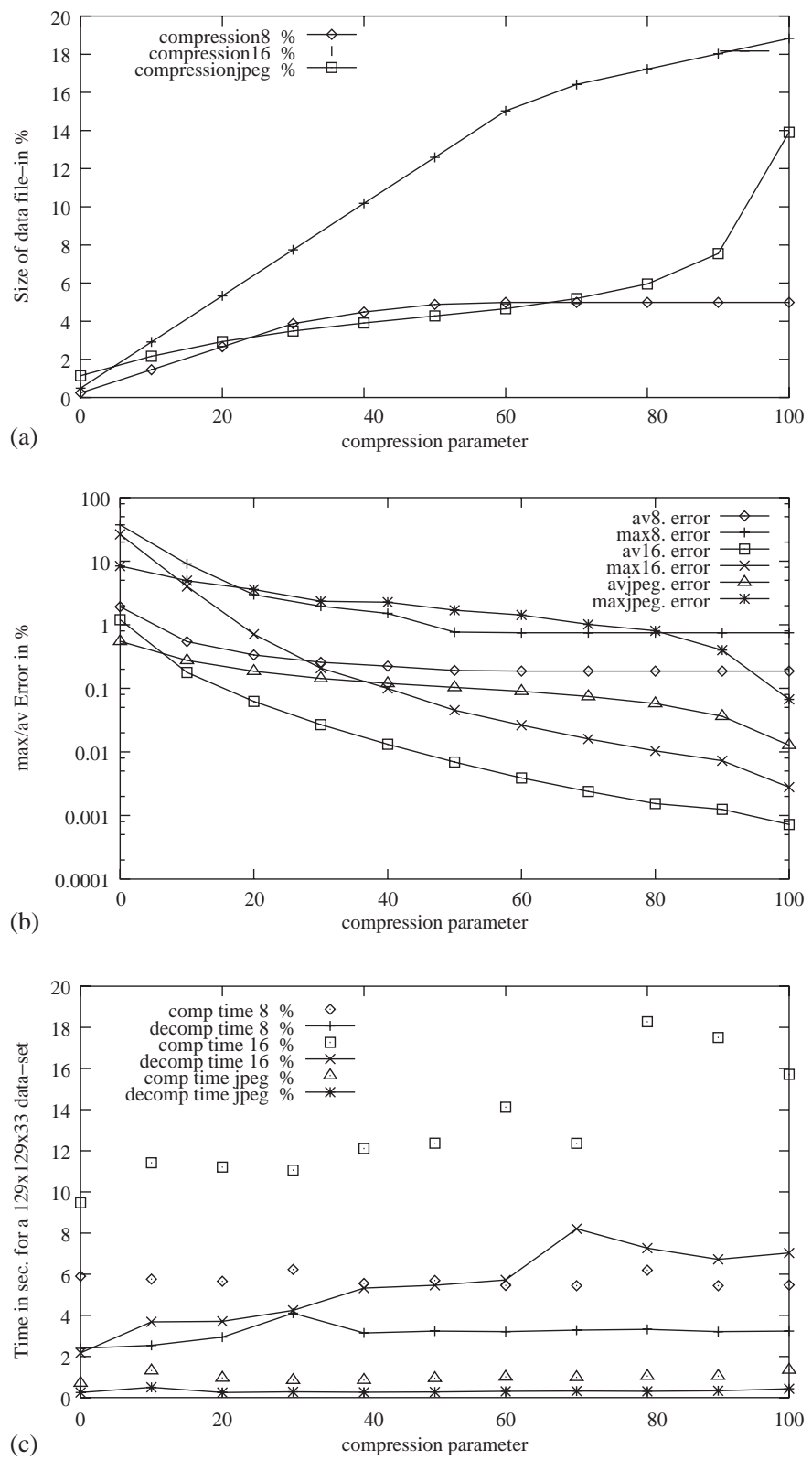

Fig. 5. Comparison of properties for 8- and 16-bit JPEG-2000 compression scheme. For better comparison we have also plotted results from 12-bit JPEG method: (a) indicates compression ratio for different values of compression parameter. In (b) maximum and averaged error connected with this compression is shown and in (c) time needed for compression and decompression is indicated.

while only compressing moderately, on the other end the MPEG-1 compression leads to extremely high compression ratios but is also generating a considerable error. In between this two compression schemes are the JPEG and JPEG-2000 compression algorithms which produces a good compression while generating only a moderate error. The size of the data set and the error of the compression can be varied over more than an order of magnitude by choosing a compression factor accordingly.

The choice of the compression scheme depends heavily on the kind of post-processing planned. For the visualization we found the MPEG-1 compressed data often being accurate enough. But for advancing tracer in a pre-computed velocity field we had to use at least a JPEG compression scheme with a high 


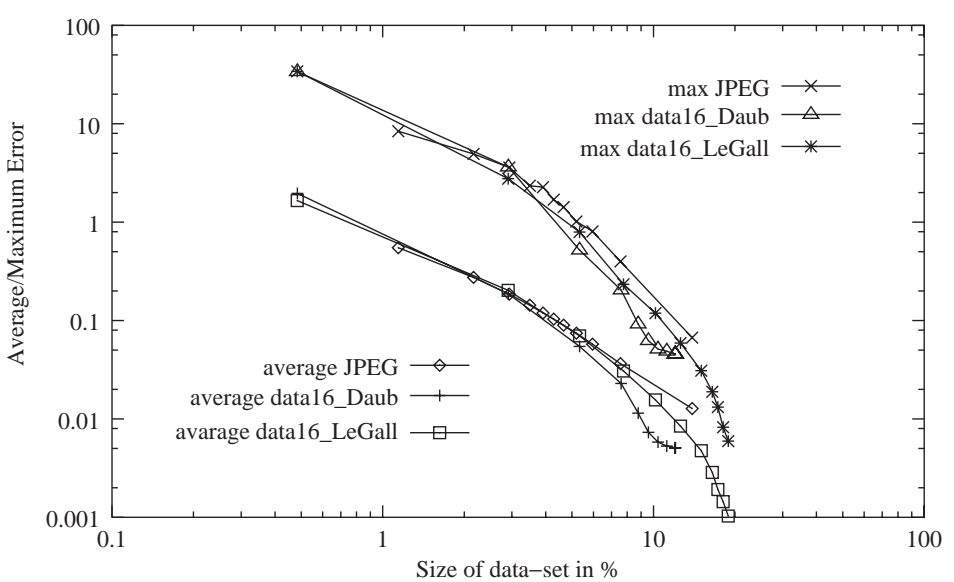

Fig. 6. Size of remaining data set in \% in dependence of averaged/maximum error generated. Both values for JPEG-2000 compression using Daubechies $(9,7)$ and Le Gall $(5,3)$ base wavelets are shown.

compression parameter in order to keep the errors in the particle paths small. For other purposes such as restarting the computation from a given time step even this accuracy may not be sufficient. At present the JPEG-2000 approach requires a lot of computing time which renders it unusable for many applications such as interactive visualization. While this is partly due to the more complex compression scheme one reason may also be the beta-test quality of the library used.

The key issue as we see it, is to have all three compression schemes easily available at the same time. This way it is possible to store e.g. every fifth time step in the MPEG-1 format for visualization while storing e.g. every 100th time step for a detailed analysis.

Therefore we present a simple wrapper library written in $C$ which gives easy access to the three compression schemes while hiding the complexity of the different interfaces.

\section{Mutils: a simple interface library to JPEG and MPEG compression}

When designing a library for scientific data storage one basically has to decide whether to make this a general tool such as $\mathrm{HDF}^{4}$ or whether to keep it as simple as possible allowing the user to easily alter the routines for his own needs. Both approaches have advantages and disadvantages and we have decided to follow the second one. Since today the majority of the codes used in CFD are written in either Fortran or $C$ it is important to provide an interface for both languages. The library itself is written in $A N S I C$ which should be available on most of today's computers. As listed in Table 2 the library itself consists of eight user callable

\footnotetext{
${ }^{4}$ HDF, Data Format, http://hdf.ncsa.uiuc.edu.
}

Table 2

Eight different user callable routines of mutils library and their purposes

\begin{tabular}{ll}
\hline mcopen & Open a mc-formatted data file \\
mcwrite & Write a data set to a mc-formatted file \\
mcread & Read a data set from a mc-formatted file \\
mcclose & Close a mc-formatted file \\
mcwinfo & Write a record with model parameters \\
mcrinfo & Read a record with model parameters \\
mcskip & Skip a data set from a mc-formatted file \\
mcback & Go one data set back on a mc-formatted file \\
\hline
\end{tabular}

routines where the first four are essential and the last four are for convenient data handling. The use of the routines, especially the parameters required, is documented in manual pages. The routine mowrite which writes one data-array expects one integer parameter for the compression on which the appropriate compression algorithm is chosen (0-100: JPEG with the same compression factor; 200 Integer16 Compression, 300 MPEG-1 Compression). We have not included the JPEG-2000 compression yet since the advantages to JPEG are only small while being compute intensive. This extensible scheme allows hiding all the other compression relevant subroutines from the user. The decompression step called with mcread also returns the compression parameter so the user knows about the quality of the data set. All three compression schemes can be arbitrarily mixed in one file. The internal structure of a single data record is shown in Fig. 7. One important feature is the storage of the length of the data stream at the beginning and at the end of the data record. This allows a fast forward and backward skipping through the data without the need of decompressing the data. The changes that had to be made to the JPEG library were marginal whereas the MPEG-1 


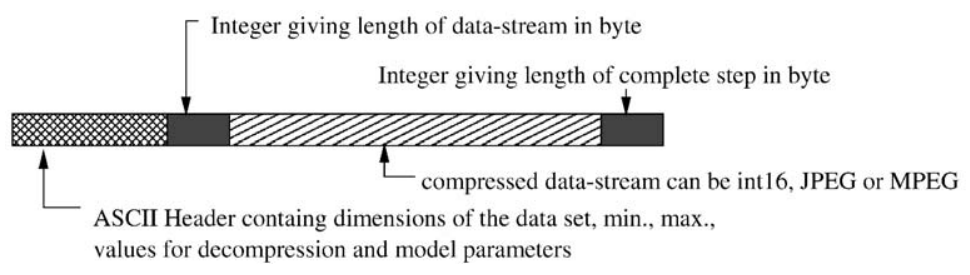

Fig. 7. Internal structure of single data record in $m c$ formatted file.

source code required substantial changes. This was caused by the fact that the MPEG- 1 code is distributed to compile as an executable program rather than a user callable library. Therefore many functions had to be changed in order to allow the multiple entrance without memory leakage. The mutils library is available upon request from the author.

\section{Conclusions}

We have investigated the properties of different lossy compression schemes for compressing data from computational fluid dynamics. Since the investigated JPEG, JPEG-2000 and MPEG compression methods are designed for image compression they may not be optimal for compressing data from numerical simulation of fluid flow. They do however provide an easily available, easily adopted and well debugged tool to compress CFD data.

Together with a simple compression scheme which stores a data-point as two ASCII characters, the investigated compression schemes compress the data in a range from $50 \%$ up to $99.7 \%$ compared to the size of the binary written data set. The compressed data are portable across different machine architectures. We have investigated the maximum and average errors connected with the compression schemes, which does correlate to the achieved compression. The processor time spent for compressing the data sets was found to be moderate for the JPEG and high for the MPEG scheme and even higher for JPEG-2000. Decompression times however proved to be much smaller for the MPEG scheme, while still being high for the JPEG-2000 scheme.

Because of the different properties of the methods we conclude that it is useful to have three methods easily available and present a simple wrapper library to access the different compression algorithms from within a CFD program.

With the market for digital image compression growing rapidly over the last years there are also new algorithms appearing. Implementations of the new MPEG-4 standards allow higher compression while maintaining a higher image quality. With the growing size of the data sets in CFD it is important to monitor these developments and to use the technology for our purposes.

\section{Acknowledgements}

I am grateful to Alexander Loddoch, one anonymous reviewer, and Greg Houseman for helpful comments.

\section{References}

Antonini, M., Barlaud, P.M., Daubechis, I., 1992. Image coding using the wavelet transform. IEEE Transactions on Image Processing 1, 205-220.

Bradley, J.N., Brislawn, C.M. 1993. Wavelet transform-vector quantization compression of super-computer ocean models. In: Proceedings of DCC '93. IEEE Computer Society Press, Silver Spring, MD, pp. 224-233.

Gall, D.L., Tabatabai, A., 1988. Sub-band coding of digital images using symmetric short kernel filters and arithmetic coding techniques. Proceedings of the IEEE International Conference on Acoustic, Speech and Signal Processing, New York, pp. 761-765.

Houseman, G.A., 1990. Boundary conditions and efficient solution algorithms for the potential function formulation of the 3-d viscous flow equation. Geophysical Journal International 100, 33-38.

ISO/IEC, 2000. Information technology_jpeg 2000 image coding system: core coding system, iso/iec jtc 1/sc 29/wg 1, iso/iec fcd 15444-1. http://www.jpeg.org/FCD15444-1.htm.

Legall, D., 1991. Mpeg - a video compression standard for multimedia applications. Communications of the ACM 34 (4), 47-58.

Lervik, J.M., Rsten, T., Ramstad, T.A., 1996. In: Lervik, J.M., Waldemar, P. (Eds.), Subband Seismic Data Compression: Optimization and Evaluation. IEEE Press, New York, NY, pp. 65-68.

Salomon, D., 2000. Data Compression. Springer, New York, NY, $821 \mathrm{p}$.

Santa-Cruz, D., Ebrahimi, T., 2000. A study of jpeg-2000 still image coding versus other standards. In: Proceedings of the $X$ European Signal Processing Conference, Vol. 2, Tampere, Finland, pp. 673-676.

Tao, H., Moorhead, R.J., 1994a. Progressive transmission of scientific data using biorthogonal wavelet transform. In: Kaufman, A.E., Bergeron, R.D. (Eds.), Proc. IEEE Visualization 94, Washington, D.C., October 1994, pp. 93-99. 
Tao, H., Moorhead, R.J., 1994b. Lossless progressive transmission of scientific data using biorthogonal wavelet bases. In: Proceedings IEEE International Conference on Image Processing, Vol. 3, pp. 373-377.

Vassiliou, A., Wickerhauser, M.V., 1997. Comparison of wavelet image coding schemes for seismic data compression. In: Akram Aldroubi, Andrew F. Laine, Michael A. Unser (Eds.), Wavelet Applications in Signal and Image Processing V, Vol. 3169, SPIE, SPIE, 27 February 1997, p. 9.

Villasenor, J.D., Ergas, R.A., Donoho, P.L., 1996. Seismic data compression using high-dimensional wavelet trans- forms. In: Storer, J.A., Cohn, M. (Eds.), Proceedings DCC '96 Data Compression Conference, Vol. 3. IEEE Computer Society Press, Silver Spring, MD, pp. 396-405.

Wilson, J., 2002. Compression of barotropic turbulence simulation data using wavelet-based lossy coding. In: Proceedings of the 2002 ASME Fluids Engineering Division Summer Meeting. FEDSM2002-31026, 2002.

Zubair, L., Sreenivasan, K.R., Wickerhauser, M.V., 1992. Characterization and compression of turbulent signals and images using wavelet-packets. In: Gadsky, T., Sirkar, S., Speziale, C. (Eds.), Studies in Turbulence. Springer, Berlin, pp. 489-513. 